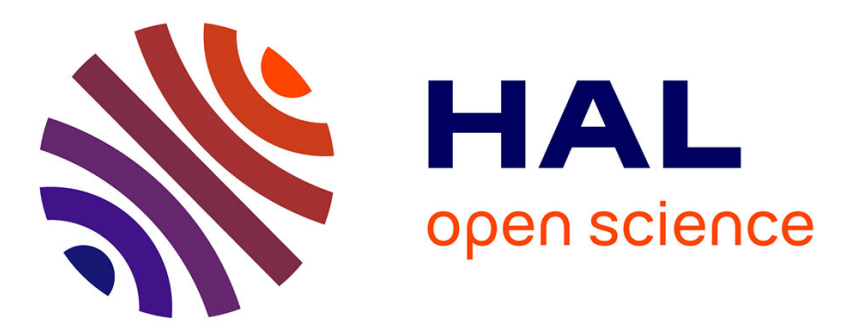

\title{
Mitigation of Carrier Frequency Offset in a Sub-THz Channel Bonding Scenario
}

\author{
Simon Bicaïs, Jean-Baptiste Doré
}

\section{To cite this version:}

Simon Bicaïs, Jean-Baptiste Doré. Mitigation of Carrier Frequency Offset in a Sub-THz Channel Bonding Scenario. IEEE International Symposium on Personal, Indoor and Mobile Radio Communications - First International Workshop on Enabling Technologies for TeraHertz Communications, Sep 2019, Istanbul, Turkey. hal-02265723

\section{HAL Id: hal-02265723 \\ https://hal.science/hal-02265723}

Submitted on 12 Aug 2019

HAL is a multi-disciplinary open access archive for the deposit and dissemination of scientific research documents, whether they are published or not. The documents may come from teaching and research institutions in France or abroad, or from public or private research centers.
L'archive ouverte pluridisciplinaire HAL, est destinée au dépôt et à la diffusion de documents scientifiques de niveau recherche, publiés ou non, émanant des établissements d'enseignement et de recherche français ou étrangers, des laboratoires publics ou privés. 


\title{
Mitigation of Carrier Frequency Offset in a Sub-THz Channel Bonding Scenario
}

\author{
Simon Bicaïs, Jean-Baptiste Doré \\ CEA-Leti, Minatec Campus, Grenoble, France \\ simon.bicais@cea.fr
}

\begin{abstract}
Wireless communications in the sub-THz bands are contemplated to achieve the soon required Tbit/s data rate. To fulfill this requirement, sub-THz transceivers aggregate several carriers, or in other words, implement channel bonding. In this paper we investigate the mitigation of the carrier frequency offset impact on channel bonding systems. In contrast to the literature, we consider that each of the carriers is corrupted by an independent random carrier frequency offset. Moreover, we do not address the carrier frequency offset estimation to compensate it but instead the optimization of the demodulation to mitigate the impact of this impairment. First, a system model is presented to describe channel bonding transceivers corrupted by carrier frequency offsets and the analytical expression of the resulting interference is outlined. Second, we pursue the analytical study for systems using waveforms with rectangular pulse-shaping. By evaluating the capacity, it is exhibited that a detection algorithm jointly demodulating the carriers could enhance the performance. Third, several detection algorithms are introduced and compared by means of numerical simulations. We show that a joint linear detection algorithm achieves a significant performance gain in comparison to a receiver demodulating independently the carriers.
\end{abstract}

\section{INTRODUCTION}

Wireless communications in sub-THz bands (100-300 $\mathrm{GHz}$ ) are emerging as a foremost solution to achieve Tbit/s data rates soon required by beyond 5G networks. Current researches on sub- $\mathrm{THz}$ communications are focused towards technology development (circuits, devices, antenna design ...). To fulfill the beyond $5 \mathrm{G}$ networks requirements, additional researches are required to design efficient and novel physical layer schemes. Traditional techniques cannot be directly transposed to sub- $\mathrm{THz}$ bands as they do not consider the unique features of Radio-Frequency (RF) architecture constraints and impairments of sub-THz systems. In particular, sub-THz communications suffer from strong phase impairments issued by high-frequency oscillators: phase noise, Carrier Frequency Offset (CFO) [1]. Regarding the targeted data rates, integrated transceivers will also have to cope with several $\mathrm{GHz}$ of band which appears to be very challenging considering current technologies for the design of embedded Analog-to-Digital Converters (ADC). From the physical layer perspective, many researches for sub- $\mathrm{THz}$ systems address the analog parallelization of multiple carriers, i.e. channel bonding, to alleviate the constraints on converters and maximize the throughput [2] [3]. The performance of these systems are severely degraded by the phase impairments. This motivates us to investigate the mitigation of the impact of CFO in a channel bonding scenario.
The performance degradation of communication systems with multiple carriers due to CFO is an extensively treated problem. Numerous algorithms have been proposed to estimate and compensate the CFO. However, most of the literature's articles consider systems aggregating several carriers in the digital domain which results in a single CFO impacting the performance. Conversely, we consider in this study the analog parallelization of multiple carriers, each of them is corrupted by an independent and randomly distributed CFO. We show that these independent CFOs lead to inter-carrier interference and severely impact the communication performance. We derive the closed-form expression of this inter-carrier interference. In contrast to state-of-the-art approaches, we do not consider the mitigation of this impairment by the estimation and compensation of CFO. In this paper, we propose and compare digital signal processing algorithms to jointly demodulate the multiple carriers in order to mitigate the impact of the CFO. Such algorithms are widely used in the domain of digital-subscriber line systems in order to mitigate cross-talk [4]. We adapt in this work theses techniques to a sub-THz channel bonding scenario. As expected a significant performance gain is demonstrated when a joint linear receiver algorithm is considered. Consequently, constraints on sub$\mathrm{THz}$ oscillators can be relaxed.

The remainder of the paper is organized as follows. Sec. II presents the model to describe sub- $\mathrm{THz}$ systems with channel bonding corrupted by CFOs and gives the analytical expression of the interference. We pursue further the analytical study in Sec. III for time-localized waveforms. Several detection algorithms to mitigate the impact of CFO are introduced and compared by simulation in Sec. IV. Eventually, Sec. V outlines potential perspectives of this work before concluding the paper.

\section{SyStem Model}

\section{A. Communication system}

We consider a coherent communication system aggregating multiple carriers as illustrated in Fig. 1. The signal to be transmitted is expressed as follows:

$$
s(t)=\sum_{k \in \mathbb{Z}} \sum_{n=1}^{N} s_{n}[k] \cdot g_{n}(t-k T), \quad t \in \mathbb{R},
$$

where $s_{n}[k] \in \mathcal{C}$ is the complex symbol sent on carrier $n \in \llbracket 1, N \rrbracket$ at time instant $k \in \mathbb{Z}$. We denote $T$ the symbol duration and $\mathcal{C}$ the symbol constellation with average energy $E_{s}$. The signals transmitted on each carrier 


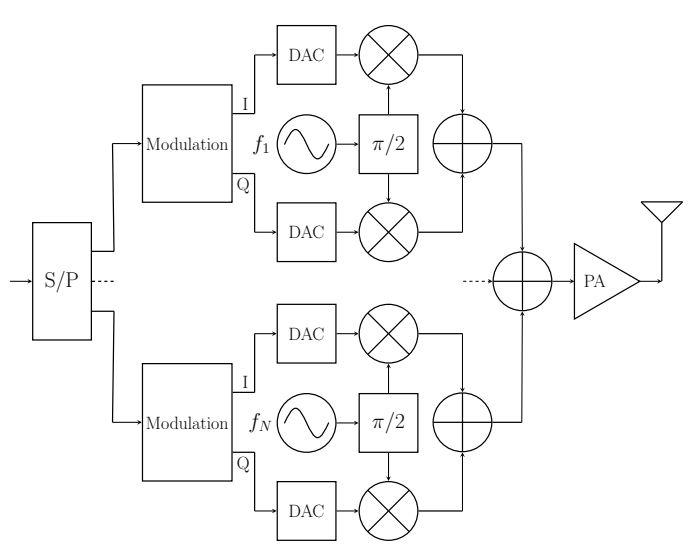

Fig. 1: Transceiver block diagram of a

are time synchronized. The communication system uses Frequency Division Multiplexing (FDM) to aggregate multiple carriers. The pulse-shaping filter on carrier $n$ satisfies

$$
g_{n}(t)=g(t) \cdot e^{j 2 \pi f_{n} t},
$$

with $g$ a common pulse-shaping filter for all carriers. The frequency of the $n$-th carrier is defined by

$$
f_{n}=f_{c}+n \cdot \Delta_{f}+\zeta_{n}
$$

where $f_{c}$ is the reference frequency, $\Delta_{f}$ is the InterCarrier Spacing (ICS) and $\zeta_{n}$ represents the random CFO of carrier $n$ - independently distributed from other carriers. The $\mathrm{CFO}$ term $\zeta_{n}$ is uniformly distributed ${ }^{1}$ as follows

$$
\zeta_{n} \sim \mathcal{U}\left[-\zeta_{\max }, \zeta_{\max }\right]
$$

The bound $\zeta_{\max }$ is the oscillator precision tolerance usually expressed either in percentage of the ICS or in parts per million (ppm) of the carrier frequency. By means of illustration, for a $200 \mathrm{GHz}$ carrier frequency and an oscillator specification of $100 \mathrm{ppm}$, which expresses an oscillator of poor quality, the maximum $\mathrm{CFO}$ is $20 \mathrm{MHz}$ which represents $4 \%$ of the ICS assuming $\Delta_{f}=500 \mathrm{MHz}$ or $20 \%$ when $\Delta_{f}=100 \mathrm{MHz}$.

\section{B. Channel model}

It has been recently confirmed by measurement campaigns [5] that the line-of-sight component prevails in sub-THz channels which can be considered as frequency flat within each of the carriers bands. The effects of the channel are assumed to be compensated ${ }^{2}$ and the receiver is synchronized. We hence study a waveform corrupted by thermal noise such that the received signal is expressed by

$$
r(t)=s(t)+\widetilde{w}(t),
$$

where $\widetilde{w}$ denotes a circularly symmetric complex Additive White Gaussian Noise (AWGN) with spectral density $N_{0}$. Sub-THz communications based on coherent

\footnotetext{
${ }^{1}$ The uniform distribution is commonly used to model the CFO based upon the specification of the oscillator precision tolerance. Nonetheless, the proposed work can be easily adapted to other distributions.

${ }^{2}$ For each carrier, the phase shift of the channel is compensated and the propagation gain is normalized.
}

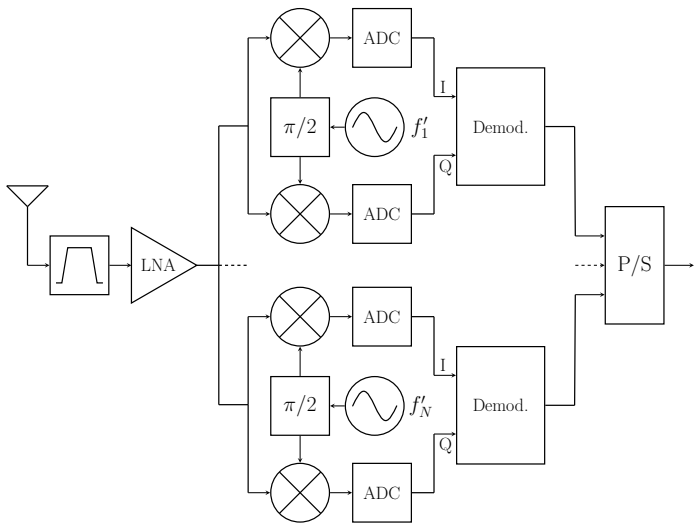

system implementing channel bonding.

receivers suffer from strong phase impairments issued by oscillators [1]: phase noise and CFO. Nevertheless, the focus of this study is on the mitigation of CFO. Therefore, the impact of $\mathrm{PN}$ on communications is not considered. Interested readers may refer to [6] for constellation optimization for phase noise or to [7] for improvement of the demodulation in the presence of phase noise.

\section{Interference expression}

We consider that the receiver is perfectly synchronized in time and frequency on each carrier. In other words, on carrier $n$, frequency $f_{n}$ is perfectly estimated such that $\zeta_{n}$ is compensated at the receiver. Our study investigates the mitigation of the interference between channels due to the independent CFOs. This work describes an openloop communication system. No bidirectional link is available to transmit back the CFOs estimations from the receiver to the transmitter in order to pre-compensate these impairments and hence to maintain carrier orthogonality. This scenario is interesting to investigate in order to show that an optimized digital processing enables to live on imperfect oscillators without correcting the different CFOs.

We now derive the analytical expression of the interference occurring on carrier $n_{0}$. The received signal is passed through the matched filter s.t.

$$
\begin{aligned}
r_{n_{0}}(t) & =\int_{-\infty}^{+\infty} r(t-\tau) \cdot g_{n_{0}}^{*}(-\tau) d \tau \\
& =\sum_{k, n} s_{n}[k] \cdot \int_{-\infty}^{+\infty} g_{n}(t-k T-\tau) \cdot g_{n_{0}}^{*}(-\tau) d \tau \\
& +\int_{-\infty}^{+\infty} \widetilde{w}(t-\tau) \cdot g_{n_{0}}^{*}(-\tau) d \tau
\end{aligned}
$$

The receiver then estimates the $k_{0}$-th symbol by sampling the signal $r_{n_{0}}$ at time instant $k_{0} T$. That is

$$
\begin{aligned}
r_{n_{0}}\left[k_{0}\right] & =r_{n_{0}}\left(k_{0} T\right), \\
& =s_{n_{0}}\left[k_{0}\right]+w_{n_{0}}+I_{n_{0}},
\end{aligned}
$$

where $w_{n_{0}} \sim \mathbb{C} \mathcal{N}\left(0, \sigma_{w}^{2}=N_{0} / T\right)$ is a band-limited complex AWGN and $I_{n_{0}}$ denotes the interference of other carriers on the one of interest. This leads to the 
expression of the Signal-to-Interference-and-Noise Ratio (SINR) on carrier $n_{0}$ given by

$$
\operatorname{SINR}_{n_{0}}=\frac{E_{s}}{\sigma_{w}^{2}+\left|I_{n_{0}}\right|^{2}} .
$$

In contrast, the Signal-to-Noise Ratio (SNR) is given by $E_{s} / \sigma_{w}^{2}$. The expression of the interference $I_{n_{0}}$ is

$$
\begin{gathered}
I_{n_{0}}=\sum_{\substack{k, n \\
n \neq n_{0}}} s_{n}[k] \cdot I_{n, n_{0}}^{k, k_{0}}, \\
I_{n, n_{0}}^{k, k_{0}}=\int_{-\infty}^{+\infty} g\left(\left(k_{0}-k\right) T+t\right) g^{*}(t) e^{j 2 \pi\left(f_{n}-f_{n_{0}}\right) t} d t .
\end{gathered}
$$

Expressing the interference with $I_{n, n_{0}}^{k, k_{0}}$ enables to delineate the impact of the waveform. Therefore, Eq. (10) can be used to evaluate the transmultiplexing response of the system.

\section{Carrier time-frequency orthogonality}

The interference term $I_{n, n_{0}}^{k, k_{0}}$ expresses the loss of orthogonality between carriers in both time and frequency. The CFOs breaks the Nyquist criterion between carriers pulse-shaping filters. A received symbol interferes with previous and future symbols from other carriers. Nonetheless, the carriers orthogonality in time is preserved when considering waveforms with a rectangular pulse-shaping, i.e. perfectly time-localized. In such case, symbols only interfere between different carriers but not between different time instants. In case of frequencylocalized carriers, only adjacent carriers interfere. In the rest of this study, we assume a strict time localization of the waveform. Discussions on the extension of this work to time-frequency localized waveforms are given in the last section.

\section{AnAlysis of Time-Localized WaVeforms}

Previously, a general model for channel bonding systems corrupted by CFOs has been described and the analytical expression of the interference has been outlined. In this section, we pursue the analytical study for systems using rectangular pulse-shaping. A matrix representation of the system model is proposed. By evaluating the capacity of the system, we show that a joint detection algorithm could achieve a performance enhancement in comparison to a system demodulating independently the corrupted carriers.

\section{A. System aggregating 2 carriers}

We first investigate a simplified scenario where the communication system aggregates two carriers and then extend the results to $N$ carriers. The signal is transmitted with a rectangular pulse-shaping, i.e.

$$
g(t)=\frac{1}{\sqrt{T}} \cdot \Pi\left(\frac{t}{T}\right) .
$$

The rectangular pulse-shaping enables us to simplify the analytical derivations since there is no interference between symbols of different time instants s.t. $\forall k \neq k_{0}, I_{n, n_{0}}^{k, k_{0}}=0$. Moreover, it has been shown

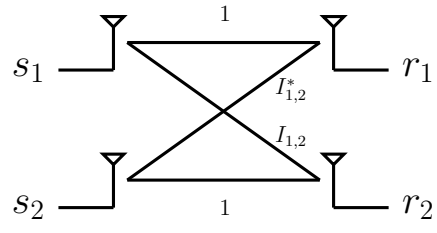

Fig. 2: Illustration of the channel model.

in [8] that rectangular waveforms, such as orthogonal frequency division multiplexing, outperform filtered waveforms, such as filter bank multi-carrier, when the communication is degraded by phase noise. The scenario is symmetric, it is thus sufficient to denote the carrier of interest $n$ and the other $\bar{n}$. We also set $\Delta_{f}=1 / T$. As there is no interference between symbols from different instants, the time index is dropped without loss of generality. From Eq. (7). The received symbol is expressed as

$$
r_{n}=s_{n}+s_{\bar{n}} \cdot I_{n, \bar{n}}+w_{n},
$$

where $w_{n} \sim \mathbb{C N}\left(0, \sigma_{w}^{2}\right)$. The interference $I_{n, \bar{n}}$ between the two carriers leads to a non-orthogonal FDM. Applying Eq. (10) to this scenario, the interference is expressed by

$$
\begin{aligned}
I_{n, \bar{n}} & =\int_{0}^{T} \frac{1}{T} \cdot e^{j 2 \pi\left(f_{n}-f_{\bar{n}}\right) t} d t, \\
& =\frac{\sin \left(2 \pi\left(f_{n}-f_{\bar{n}}\right) T\right)}{2 \pi\left(f_{n}-f_{\bar{n}}\right) T}+j \frac{1-\cos \left(2 \pi\left(f_{n}-f_{\bar{n}}\right) T\right)}{2 \pi\left(f_{n}-f_{\bar{n}}\right) T}
\end{aligned}
$$

with $\left(f_{n}-f_{\bar{n}}\right)=\Delta_{f}+\zeta_{n}-\zeta_{\bar{n}}$. It is worth mentioning that the above formula remains valid for any $N$ and that $I_{\bar{n}, n}=I_{n, \bar{n}}^{*}$. For small frequency offsets $\zeta_{n} \ll \Delta_{f}$, the interference may be estimated with a 1st-order approximation by

$$
I_{n, \bar{n}} \simeq \operatorname{sign}\left(f_{n}-f_{\bar{n}}\right) \cdot\left(\zeta_{n}-\zeta_{\bar{n}}\right) \cdot T .
$$

The interference is here approximated by the ratio of CFO over the ICS. This further supports the expression of $\zeta_{\max }$ as a fraction of the ICS, accordingly we hereafter use the normalized maximum $\mathrm{CFO} \zeta_{\max } \cdot T$. As expected, the stronger $\zeta_{\max } \cdot T$, the stronger the interference. It follows from Eq. (12) that the channel can be expressed with a matrix notation as follows

$$
\begin{aligned}
\boldsymbol{r} & =H \boldsymbol{s}+\boldsymbol{w} \\
{\left[\begin{array}{l}
r_{1} \\
r_{2}
\end{array}\right] } & =\left[\begin{array}{cc}
1 & I_{1,2}^{*} \\
I_{1,2} & 1
\end{array}\right]\left[\begin{array}{l}
s_{1} \\
s_{2}
\end{array}\right]+\boldsymbol{w},
\end{aligned}
$$

where $r \in \mathbb{C}^{2}, H \in \mathbb{C}^{2 \times 2}$ and $s \in \mathcal{C}^{2}$. Here, $w=\left(w_{1}, w_{2}\right)$ and $w_{n} \sim \mathcal{N}\left(0, \sigma_{w}^{2}\right)$. This channel model is depicted in Fig. 2 and corresponds to the complex baseband vector notation of a single-user MIMO link. The eigenvalues of the channel matrix $H$ are $\lambda_{1}=1-\left|I_{1,2}\right|$ and $\lambda_{2}=1+\left|I_{1,2}\right|$. For the considered channel and with $\zeta=\left(\zeta_{1}, \zeta_{2}\right)$, the capacity is given by

$$
C(\boldsymbol{\zeta})=\sum_{n=1}^{N} \frac{1}{T} \log _{2}\left(1+\mathrm{SNR} \cdot \lambda_{n}(\boldsymbol{\zeta})\right) .
$$




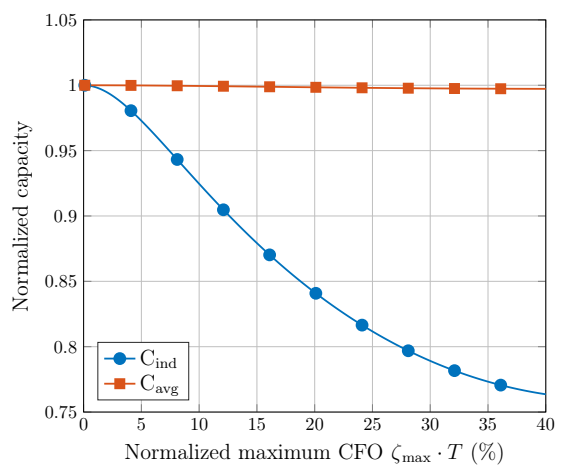

Fig. 3: Capacities with interference between carriers.

It is worth mentioning that the interference term $I_{1,2}$ is only function of the sum $\zeta_{\text {tot }}=\zeta_{n}+\zeta_{\bar{n}}$ and so is the capacity. Then, for a given bound $\zeta_{\max }$ the average value of the capacity is given by

$$
C_{\text {avg }}\left(\zeta_{\max }\right)=\int_{-2 \zeta_{\max }}^{+2 \zeta_{\max }} C(u) f_{\zeta_{\text {tot }}}(u) d u,
$$

where $f_{\zeta_{\text {tot }}}$ denotes the probability density function of $\zeta_{\text {tot }}=\zeta_{n}+\zeta_{\bar{n}}$ defined by the linear piecewise expression

$f_{\zeta_{\mathrm{tot}}}(u)= \begin{cases}\frac{1}{2 \zeta_{\max }}\left(1+\frac{u}{2 \zeta_{\max }}\right), & \text { if }-2 \zeta_{\max } \leq \zeta<0, \\ \frac{1}{2 \zeta_{\max }}\left(1-\frac{u}{2 \zeta_{\max }}\right), & \text { if } 0 \leq \zeta \leq 2 \zeta_{\max }, \\ 0, \text { otherwise, } & \end{cases}$

Fig. 3 depicts the average capacity $C_{\text {avg }}$ as function of $\zeta_{\max } \cdot T$ normalized to the capacity of the system without CFO. Moreover, the capacity $C_{\text {ind }}$ is also illustrated in Fig. 3 which is defined by

$$
\begin{gathered}
C_{\text {ind }}\left(\zeta_{\max }\right)=\int_{-2 \zeta_{\max }}^{+2 \zeta_{\max }} C(u) f_{\zeta_{\text {tot }}}(u) d u, \\
C(\boldsymbol{\zeta})=\sum_{n=1}^{N} \frac{1}{T} \log _{2}\left(1+\operatorname{SINR}_{n}(\boldsymbol{\zeta})\right) .
\end{gathered}
$$

The latter equation corresponds to the capacity of a system demodulating the carriers independently. The interference is then perceived as an unknown Gaussian noise. It is worth mentioning that the capacity $C_{\mathrm{avg}}$ decreases slightly while $C_{\text {ind }}$ decays rapidly as the maximum CFO increases. These observations imply that the interference bears useful information that could be exploited by a joint demodulation of carriers to improve the system performance.

\section{B. Extension to $N$ carriers}

The channel model described in Eq. (15) may be extended to $N$ carriers provided that the pulse-shaping filter remains rectangular. The channel is expressed in this case by

$$
\boldsymbol{r}=H \boldsymbol{s}+\boldsymbol{w},
$$

where $\boldsymbol{r} \in \mathbb{C}^{N}, H \in \mathbb{C}^{N \times N}$ and $\boldsymbol{s} \in \mathcal{C}^{N}$. Here, $\boldsymbol{w}=\left(w_{1}, \cdots, w_{n}\right)$ and $w_{n} \sim \mathcal{N}\left(0, \sigma_{w}^{2}\right)$. It should be noted that the expressions of the interference or capacities remains valid for any $N$. The channel matrix is defined by $H=\left(I_{i, j}\right)_{1 \leq i, j \leq N}$, where $I_{i, j}$ represents the interference between carrier $i$ and $j$ defined in Eq. (13). It is worth mention that in Eq. (13), $I_{i, i}=1$. This model has been employed for crosstalk cancellation in [9] where a Tomlinson-Harashima precoding scheme is used to improve the throughput of the channel. Similarly to Eq. (15), the channel model in Eq. (21) corresponds to a single-user MIMO link. Therefore, the conventional MIMO techniques may be used to improve the performance in the treated scenario. The following section investigates the use of a joint detection algorithm to improve performance of a system aggregating multiple carriers with a rectangular pulse-shaping and corrupted by CFOs.

\section{Joint Demodulation Algorithms}

In this section, we present and compare detection algorithms with a joint demodulation of carriers in order to mitigate the impact of CFOs. A joint detection algorithm is expected to enhance performance by decoupling the inter-carrier interferences to demodulate the sent symbols.

\section{A. Algorithms description}

1) Independent: We first introduce the receiver algorithm demodulating independently the carriers. This enables us to set a reference for comparison with the further introduced joint algorithms. This receiver, illustrated in Fig. 1, demodulates each of the symbol independently. As the sent symbols are considered equiprobable, it is known [10] that the optimum decision rule - minimizing the probability of error - is the Maximum Likelihood (ML) detection criterion. For carrier $n$ at a given instant, the demodulated symbol $\widehat{s}_{n}$ is hence given by

$$
\widehat{s}_{n}=\underset{s \in \mathcal{C}}{\arg \max } p\left(r_{n} \mid s\right),
$$

where $p\left(r_{n} \mid s\right)$ is the channel likelihood function. This receiver treats the inter-carrier interference as noise. The channel being modeled as an AWGN one, the detection criterion is easily shown to be expressed upon the Euclidean distance by

$$
\widehat{s}_{n}=\underset{s \in \mathcal{C}}{\arg \min }\left\|r_{n}-s\right\|_{2} .
$$

This decision rule entirely defines the independent detection algorithm.

2) Joint $M L$ : From now on, symbols $s$ of all carriers at a given instant are demodulated jointly. Regarding the system model defined in Eq. (21), it is known that the optimal decision rule for equiprobable symbol is a joint ML detection criterion. This is expressed by

$$
\widehat{\boldsymbol{s}}=\underset{\boldsymbol{s} \in \mathcal{C}^{N}}{\arg \min } p(\boldsymbol{r} \mid \boldsymbol{s}, H) .
$$

It can be easily checked that the joint channel likelihood function is a multi-dimensional normal distribution such that the detection criterion is based upon the multidimensional Euclidean distance and is given by

$$
\widehat{\boldsymbol{s}}=\underset{\boldsymbol{s} \in \mathcal{C}^{N}}{\arg \min }\|\boldsymbol{r}-H \boldsymbol{s}\|_{2} .
$$


Nonetheless, the joint ML detection algorithm cannot be implemented in practical communication systems - with limited memory, latency and computational resources. This detector involves the evaluation of the objective function $\|\boldsymbol{r}-H \boldsymbol{s}\|_{2}$ for all $s \in \mathcal{C}^{N}$ the possible transmitted symbols. The minimization over $\mathcal{C}^{N}$ entails an algorithm complexity exponentially increasing with $N$.

3) Linear: The complexity of a linear receiver is the complexity of inverting a matrix of dimensions $N \times N$. The linear receiver relies on the decoupling of the inter-carrier interferences. This is achieved by the equalization of $H$. We consider here a Zero Forcing (ZF) equalization criterion. In others words, received symbols are multiplied by filter $W=\left(H^{H} H\right)^{-1} H^{H}$, the pseudo inverse of $H$. We denote $\widetilde{\boldsymbol{s}}(\boldsymbol{r})=W \boldsymbol{r}$. Then, the symbols are estimated independently according to the following decision rule

$$
\widehat{s}_{n}=\underset{s \in \mathcal{C}}{\arg \min }\left\|\widetilde{s}_{n}-s\right\|_{2} .
$$

The complexity of computing $W$ is roughly cubic in $N$ while estimating $\widehat{s}$ from $\widetilde{s}$ is realized in linear time with $N$.

4) Linear with precoding: We present here the combination of the linear receiver with an Hadamard precoding. Many MIMO techniques are based upon precoding schemes such as Singular Value Decomposition (SVD) achieving the MIMO channel capacity. However, SVD is not possible here as we consider open loop systems. We introduce here a fixed Hadamard precoding scheme in order to average the interference over all carriers and hence to enhance performance. At the transmitter side, modulated symbols are multiplied by precoding matrix, i.e. $P s$, before being transmitted. Matrix $P$ is the $N \times N$ Hadamard matrix normalized by $1 / \sqrt{N}$. At the receiver side, symbols are first filtered by $W$ to decouple the interferences and then multiplied by $P^{-1}$ to perform the decoding. This is expressed by denoting $\widetilde{\boldsymbol{s}}(\boldsymbol{r})=P^{-1} W \boldsymbol{r}$. It follows that the decision rule is

$$
\widehat{s}_{n}=\underset{s \in \mathcal{C}}{\arg \min }\left\|\widetilde{s}_{n}-s\right\|_{2} .
$$

The complexity of this algorithm, in comparison to the linear receiver, is increased only by a matrix multiplication. However, Fast Walsh-Hadamard transform could be implemented to reduced complexity. It is worth mentioning that the precoding scheme at the transmitter is fixed, such that the receiver know the values ${ }^{3}$ of $P^{-1}$ to perform the demodulation. Let us now evaluate and compare the performance of these detection algorithms by simulation.

\section{B. Simulation results}

In this section, we provide numerical simulations evaluating the performance of the presented detection algorithms. Fig. 4 presents the Bit Error Rate (BER) performance of a system aggregating 16 carriers with a

\footnotetext{
${ }^{3}$ The inverse of the considered Hadamard precoding matrix is its transpose, i.e. $P^{-1}=P^{\top}$, and is hence trivially obtained.
}

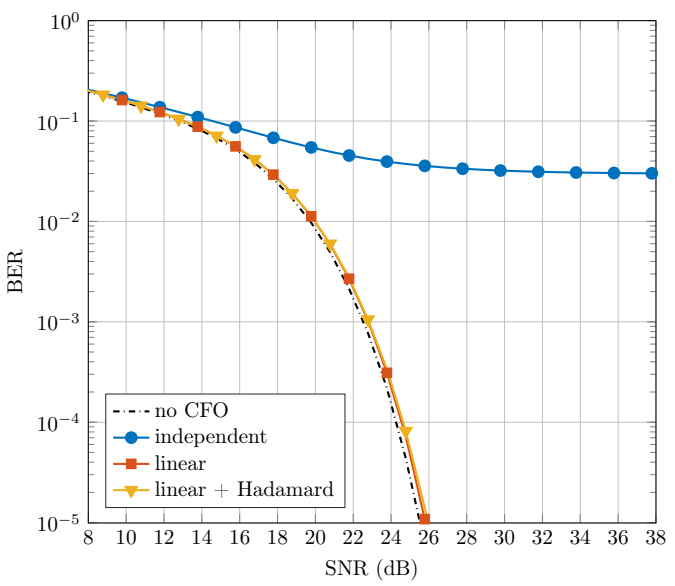

(a) $\zeta_{\max } \cdot T=10 \%$.

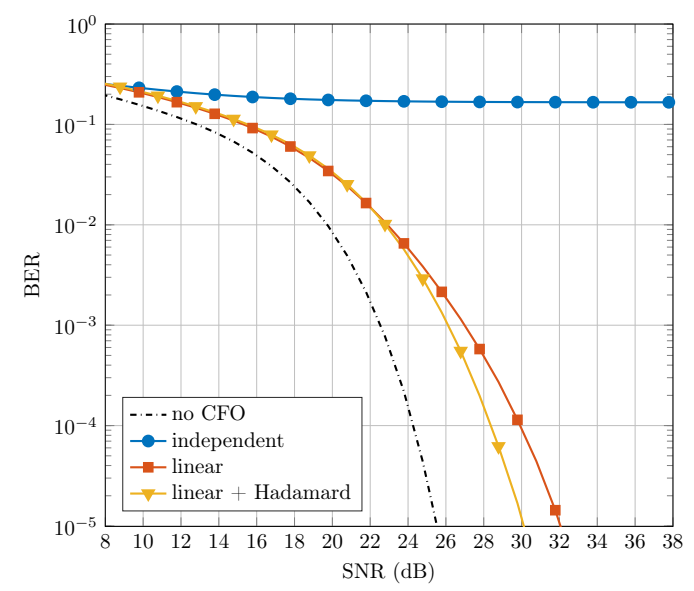

(b) $\zeta_{\max } \cdot T=30 \%$.

Fig. 4: Performance of the different detection algorithms for a system aggregating 16 carriers with a 64-QAM.

64 points Quadrature Amplitude Modulation (QAM) for the different detection algorithms. In particular, Fig. 4a depicts the simulation results with a normalized maximum $\mathrm{CFO} \zeta_{\max } \cdot T=10 \%$ and Fig. $4 \mathrm{~b}$ the results for $\zeta_{\max } \cdot T=30 \%$. First, it should be noticed that the performance of the independent detection receiver are significantly degraded even for small values of $\zeta_{\max } \cdot T$. Second, for $\zeta_{\max } \cdot T<10 \%$, the performance of the other detections algorithms are very similar and close to the performance of the system without interference. Third, for strong values of $\zeta_{\max } \cdot T-e . g .30 \%$ - the linear receiver combined with a Hadamard precoding outperforms the other detections algorithms. We can conclude upon these results for systems aggregating multiple carriers that a significant performance gain may be realized with a linear joint detection algorithm even for high value of independent CFO. To further support this assessments, Fig. 5 presents for a 128 carriers system with a 256-QAM the simulated uncoded BER as a function of $\zeta_{\max } \cdot T$ with a fixed value of SNR. Fig. 5 confirms the aforementioned properties and also exhibits the possibility to relax constraint on local oscillators with optimized digital signal processing. A feedback from the 


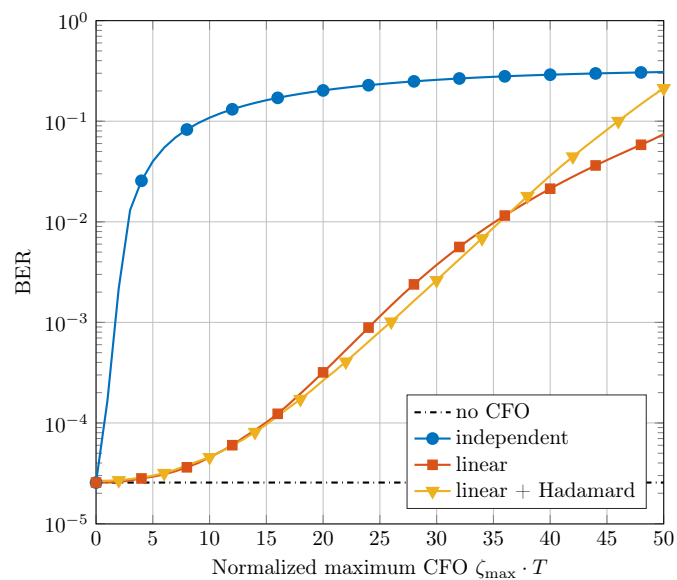

Fig. 5: Performance of the detection algorithms for a 128 carriers system and a 256-QAM with SNR $=30 \mathrm{~dB}$.

receiver is not required to compensate at the transmitter the CFOs and maintain carrier orthogonality. To illustrate this statement, let us derive a numerical application. The sub- $\mathrm{THz}$ channel bandwidth are contemplated to be of the order of the GHz. Considering a $1 \mathrm{GHz}$ band, a maximum normalized CFO of $10 \%$ represents a $100 \mathrm{MHz}$ bound or a $500 \mathrm{ppm}$ specification for a 200 $\mathrm{GHz}$ oscillator. As observed on Fig. 5 if $\zeta \mathrm{max} \cdot T$ is less than $10 \%$ then the performance loss caused by the CFOs is very small with a joint detection algorithm and could be considered tolerable.

\section{Perspectives \& Conclusion}

\section{A. Perspectives and filtered waveforms}

We have limited the study to waveforms with rectangular pulse-shaping. It has been shown that a joint linear detection algorithm achieves a performance gain and enables to relax constraints on RF oscillators. The perspectives of the presented work may consist in evaluating other detection algorithms such as sphere decoders for instance. Furthermore, we contemplate to extend the study to the joint detection of filtered waveforms. As previously discussed, for filtered waveforms the interference induced by the CFO occurs between symbols of different carriers and time instants. The system model cannot be described as straightforwardly with a matrix notation. Subsequently, the joint detection algorithms have to demodulate jointly the symbols in time and frequency. Such joint detection algorithms could relax the constraints on guard bands between channels and hence improve the spectral efficiency. Interference cancellation in multi-carrier systems with time-frequency localized waveforms is an on-going research topic and remains an open problem, for a detailed study see [11].

\section{B. Conclusion}

In this paper we have addressed the mitigation of the CFO impact in sub-THz channel bonding systems. In contrast to the literature, this work does not investigate the estimation and compensation of a single CFO. Conversely, we have considered an open-loop system aggregating multiple carriers each of them being corrupted by an independent randomly distributed CFO. Detection algorithms have been studied to jointly demodulate the carriers even with strong CFOs. First, we have described a general system model for channel bonding transceivers. An analytical expression of the interference induced by the CFOs has been derived. Second, we have pursued an analytical analysis for waveforms with rectangular pulse-shaping. Evaluating the capacity of the system corrupted by CFOs has exhibited the potential performance enhancement achievable by a joint detection algorithm. Third, several detection algorithms have been presented and compared. We have shown by numerical simulations that a joint linear detection algorithms achieve a significant performance gain, in comparison to a system demodulating the carriers independently. The BER degradation remains reasonable when a joint detection algorithm is used even for large oscillator precision tolerances. This works shows that optimizing digital signal processing enables to relax constraint on the local oscillators.

\section{REFERENCES}

[1] M. Voicu, D. Pepe, and D. Zito, "Performance and Trends in Millimetre-Wave CMOS Oscillators for Emerging Wireless Applications," International Journal of Microwave Science and Technology, vol. 2013, p. 6, 2013.

[2] C. Wang, B. Lu, C. Lin, Q. Chen, L. Miao, X. Deng, and J. Zhang, "0.34-THz Wireless Link Based on High-Order Modulation for Future Wireless Local Area Network Applications," IEEE Transactions on Terahertz Science and Technology, vol. 4, no. 1, pp. 75-85, Jan 2014.

[3] A. Kanno, K. Inagaki, I. Morohashi, T. Sakamoto, T. Kuri, I. Hosako, T. Kawanishi, Y. Yoshida, and K. ichi Kitayama, "20$\mathrm{Gb} / \mathrm{s}$ QPSK W-band $(75-110 \mathrm{GHz})$ wireless link in free space using radio-over-fiber technique," IEICE Electronics Express, vol. 8, no. 8, pp. 612-617, 2011.

[4] M. Dngen, "Crosstalk Mitigation Techniques for Digital Subscriber Line Systems," Ph.D. dissertation, Technischen Universitt Hamburg-Harburg, 2016.

[5] L. Pometcu and R. D'Errico, "Characterization of Sub-THz and mmWave Propagation Channel for Indoor Scenarios," in 12th European Association on Antennas and Propagation (EurAAP 18), Apr 2018.

[6] R. Krishnan, A. G. i Amat, T. Eriksson, and G. Colavolpe, "Constellation optimization in the presence of strong phase noise," IEEE Transactions on Communications, vol. 61, no. 12, pp. 5056-5066, December 2013.

[7] S. Bicaïs, J.-B. Doré, and J.-L. Gonzalez Jimenez, "On the Optimum Demodulation in the Presence of Gaussian Phase Noise," in 2018 International Conference on Telecommunications (ICT), June 2018.

[8] V. Moles-Cases, A. A. Zaidi, X. Chen, T. J. Oechtering, and R. Baldemair, "A comparison of OFDM, QAM-FBMC, and OQAM-FBMC waveforms subject to phase noise," in 2017 IEEE International Conference on Communications (ICC), May 2017, pp. $1-6$.

[9] G. Ginis and J. M. Cioffi, "A multi-user precoding scheme achieving crosstalk cancellation with application to DSL systems," in Conference Record of the Thirty-Fourth Asilomar Conference on Signals, Systems and Computers (Cat. No.00CH37154), vol. 2, Oct 2000, pp. 1627-1631 vol.2.

[10] J. Proakis, Digital Communications 5th Edition, ser. McGrawHill series in electrical and computer engineering : communications and signal processing. McGraw-Hill, 2007.

[11] P. Chevalier, R. Chauvat, and J. Delmas, "Enhanced Widely Linear Filtering to Make Quasi-Rectilinear Signals Almost Equivalent to Rectilinear Ones for SAIC/MAIC," IEEE Transactions on Signal Processing, vol. 66, no. 6, pp. 1438-1453, March 2018. 